\title{
De Novo sequencing and transcriptome analysis for Tetramorium bicarinatum: a comprehensive venom gland transcriptome analysis from an ant species
}

Wafa Bouzid ${ }^{1}$, Marion Verdenaud ${ }^{2}$, Christophe Klopp $^{3}$, Frédéric Ducancel$^{2}$, Céline Noirot $^{3}$ and Angélique Vétillard ${ }^{1 *}$

\begin{abstract}
Background: Arthropod venoms are invaluable sources of bioactive substances with biotechnological application. The limited availability of some venoms, such as those from ants, has restricted the knowledge about the composition and the potential that these biomolecules could represent. In order to provide a global insight on the transcripts expressed in the venom gland of the Brazilian ant species Tetramorium bicarinatum and to unveil the potential of its products, high-throughput approach using Illumina technology has been applied to analyze the genes expressed in active venom glands of this ant species.
\end{abstract}

Results: A total of 212,371,758 pairs of quality-filtered, 100-base-pair Illumina reads were obtained. The de novo assemblies yielded 36,042 contigs for which 27,873 have at least one predicted ORF among which $59.77 \%$ produce significant hits in the available databases. The investigation of the reads mapping toxin class revealed a high diversification with the major part consistent with the classical hymenopteran venom protein signature represented by venom allergen (33.3\%), followed by a diverse toxin-expression profile including several distinct isoforms of phospholipase $A_{1}$ and $A_{2}$, venom serine protease, hyaluronidase, protease inhibitor and secapin. Moreover, our results revealed for the first time the presence of toxin-like peptides that have been previously identified from unrelated venomous animals such as waprin-like (snakes) and agatoxins (spiders and conus).

The non-toxin transcripts were mainly represented by contigs involved in protein folding and translation, consistent with the protein-secretory function of the venom gland tissue. Finally, about $40 \%$ of the generated contigs have no hits in the databases with $25 \%$ of the predicted peptides bearing signal peptide emphasizing the potential of the investigation of these sequences as source of new molecules. Among these contigs, six putative novel peptides that show homologies with previously identified antimicrobial peptides were identified.

Conclusions: To the best of our knowledge, this work reports the first large-scale analysis of genes transcribed by the venomous gland of the ant species T. bicarinatum and helps with the identification of Hymenoptera toxin arsenal. In addition, results from this study demonstrate that de novo transcriptome assembly allows useful venom gene expression analysis in a species lacking a genome sequence database.

Keywords: Tetramorium bicarinatum, Social hymenoptera, Ant, Venom glands, Venom toxins, Hymenopteran allergens, de novo assembly, New generation sequencing, Illumina technology

\footnotetext{
* Correspondence: angelique.vetillard@univ-jfc.fr

'Venoms and Biological Activities Laboratory, EA 4357, PRES-University of

Toulouse, Jean-François Champollion University Center, Albi, France

Full list of author information is available at the end of the article
} 


\section{Background}

The biodiversity and specificity of animal venom compounds make them an uncommon and invaluable source from which pharmacological and therapeutic agents can be established [1]. In Hymenoptera, the venom gland shows plasticity associated with organism life history diversification and venom compounds have evolved as important weapons used for colony or individual defense [2]. Unlike other venoms such as from snakes, hymenopteran stings are generally not lethal causing mainly inflammatory and/or immunological reactions despite that some venoms from solitary Hymenoptera have evolved to cause paralysis to permit egg laying on their arthropod hosts [2]. Toxin peptides from social Hymenoptera follow similar general features with short and linear polycationic peptides, responsible of cell lysis, hemolysis, histamine release and antimicrobial actions [3,4]. Current knowledge of venom proteins and peptides involved in these processes is rather limited to stinging model species from wasps, bees, ants [5] or solitary endoparasitoid wasps [6]. Hence, little data are available from other groups especially from ants in spite of the astonishing taxonomic diversification in this insect group [5,7]. Unlike stings from social bees and wasps that are solely used for defense, those from ants have additional functions as prey capture, aggregation and social communication which implies more diverse venom compounds. In addition, composition of venoms from ants varies significantly between the different ant subfamilies with probably unique venom components specific to each group which warrants their investigation [7]. This fact has been approved until recently by a comprehensive study of the venom gland transcriptome of the giant ant Dinoponera quadriceps that revealed species-specific toxin diversification [8].

In addition to biochemical investigation, 'Venomics' involving cutting-edge transcriptomics, proteomics and high-throughput venom peptide characterization technologies are emerging projects aiming at unraveling animal venom complexity for both fundamental and practical aspects $[9,10]$. Given that venom proteins/peptides are produced in dedicated glands, transcriptome sequencing has proven to be an effective approach to identifying the expressed toxin genes. This is of particular interest for venoms that are difficult to sample such as mandibular venoms or for animals producing limited venom amounts. The latter case applies in particular to ants, which are, due to their tiny body and venom apparatus size, difficult to screen by analytical investigation unless laborious venom sac dissection is undergone with many thousands of individuals sacrificed [11].

Our study species Tetramorium bicarinatum [Hymenoptera: Formicidae] was chosen because of the diversity of its biotopes and also because this ant genus is more primitive than other highly derived genus (such as the massively studied genus Solenopsis). Hence, it is considered to produce more protein venom [12]. In a previous study, we generated Expressed Sequence Tags (ESTs) from the venom-gland transcriptome of this species, however the study relied on Sanger sequencing, generating important, but ultimately limited data [13]. For non-model organisms lacking defined genomes such as our studied species, de novo assembly is typically required for downstream RNASeq analyses [14].

In the current study, we characterized the transcriptional expression in the venom gland cells of the ant species $T$. bicarinatum using next generation illumina sequencing technology. Along with de novo assembly and transcriptome annotation, analysis of global patterns of gene expression and functional categorization were performed. In addition, features of some relevant putative toxin candidates are discussed.

\section{Results and discussion}

\section{Illumina NGS and read assembly}

The gene expression profile of Tetramorium bicarinatum venom glands were deduced from mRNA samples of ant whole body and venom gland tissues using the Illumina sequencing approach. After the sequencing quality filtering step, a total of 424,743,516 of 100-basepair Illumina reads were obtained for both samples (Table 1). The de novo assemblies yielded 37,818 contigs for the two libraries (venom glands and ant carcasses) among which 33,241 contigs were shared by both tissues (Figure 1). All unassembled sequencing reads, which accounted for $25 \%$ for the ant library and $50 \%$ for the venom gland library, were excluded from our investigation. As the aim of this study was to profile venom transcriptome, we mainly focus on the 36,042 contigs recovered from the venom gland library. The contigs recorded from the ant whole body sample were used to

\begin{tabular}{|c|c|}
\hline Total number of reads & $424,743,516$ \\
\hline Total base pairs & $42,474,351,600$ \\
\hline Average read length & $100 \mathrm{bp}$ \\
\hline Total number of contigs & 37,818 \\
\hline $\begin{array}{l}\text { Number of contigs having mapped reads from } \\
\text { ant library }\end{array}$ & 35,017 \\
\hline $\begin{array}{l}\text { Number of contigs having mapped reads from } \\
\text { venom gland library }\end{array}$ & 36,042 \\
\hline $\begin{array}{l}\text { Number of unigenes from gland library (based } \\
\text { on the annotation) }\end{array}$ & 28,294 \\
\hline Mean length of contigs & $1839 \mathrm{bp}$ \\
\hline $\begin{array}{l}\text { Total number of sequences with at least one } \\
\text { transcribed sequence }\end{array}$ & 27,873 \\
\hline
\end{tabular}




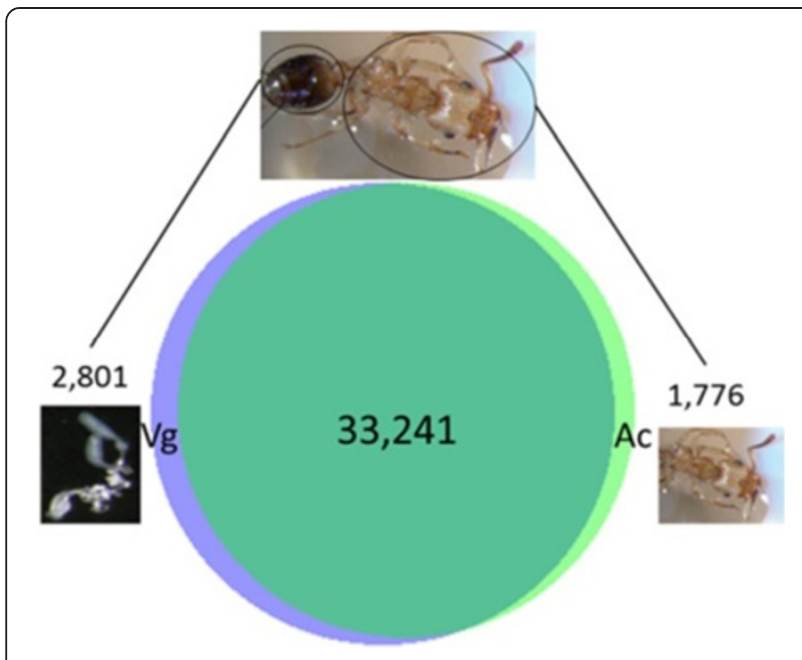

Figure 1 Number of Illumina contigs generated by de novo assembly from Tetramorium bicarinatum. $\mathrm{Vg}$ refers to venom glands and Ac to ant carcasses. 33,241 contigs were shared by both samples and 2,801 contigs have only been detected in venom glands.

assess differentially expressed genes. Among these detected transcripts (contigs), 27,873 have at least one predicted ORF with $59.77 \%$ producing significant hits in the available databases. Gene ontology (GO) functional categorization of the annotated contigs from T. bicarinatum venom glands is provided on Figure 2. The GO analysis in relation to molecular functions revealed that the largest number of transcripts was related to protein binding, followed by catalytic activities and at a lower rate to structural protein and transporter activities (Figure 2A). Analysis of the biological processes revealed two dominant major gene categories of cellular and metabolic processes. The following categories are related to biological regulation, localization, response to stimulus, biogenesis and signaling (Figure 2B).

In order to assess differentially expressed genes in the venom glands, we used the previously described DESeq approach implemented in $\mathrm{R}$ package which allows investigation of differential expressed transcripts from high-throughput data without replicates [15]. Regarding a likely important expression level variation between the two tested samples, the analysis failed to give workable results. This bias could be linked to the necessary preamplification of the venom gland sample prior to library construction compared to ant whole body sample from which extracted RNA quantity was sufficient to be directly processed to cDNA library construction (see Methods section). To bypass this bias probably originating from the amplification step, we set an arbitrary $10^{3}$ fold expressionthreshold to the 37,818 contigs obtained from both sequenced samples. Due to a lower quality of the sequencing of the read2, we decided to perform our analysis using only the read 1 for both samples. In total, we generated a list of 502 contigs that are 1000 fold more expressed in the venom gland, that we called the 'venom-gland overexpressed' transcripts. Analysis of the functional diversification among this group revealed a significant abundance (77\%) of contigs that have no hits in the databases (Figure 3). On the other hand, contigs that matched predicted toxins and non-toxin proteins, accounted for $11 \%$ and $3 \%$, respectively. The remaining group of $9 \%$ consisted of uncharacterized compounds (Figure 3). Moreover, about 235 contigs have significant hit with available bacterial sequences. These sequences have not been eliminated given that their low number does not affect the overall assemblage quality. The distribution of the possible bacterial community cohabiting with $T$. bicarinatum according to their contig number is provided in (Additional file 1: Figure S1).

\section{Cluster relevant to cellular functions}

Among the 502 'over-expressed' transcripts, 3\% clusters presented significant hits in the databases involved in various cellular functions (Figure 3). The most relatively high expressed transcripts of this category are those matching methyl-transferases (61\%) having transcription co-activator activities. This finding is in agreement with previous work showing that 'gene transcription protein' are the most abundant cellular transcripts reflecting the functional feature of the specialized tissues such as venom gland cells [16]. In addition, significant transcripts (4\%) matched the glucose dehydrogenase involved in carbohydrate metabolism. This enzyme, that belongs to a family of oxido-reductases, has been identified in a recent work based on genome mining and proteomic analysis and was described as a novelty in insect venoms [6].

\section{Cluster related to venom toxins}

Venoms from social Hymenoptera are important defensive weapons in which the most common components are low molecular proteins recognized as important allergens and resulting in an IgE-mediated reaction [17]. The investigation of the reads mapping to a toxin class that accounted for $11 \%$ of the 'over-expressed' genes revealed a high diversification with the major part represented by hymenopteran allergens (Additional file 2: Table S1). As can be seen in Figure 3, venom allergens 3 and 5 have been found among the main 'over-expressed' transcripts in the toxin-like group $(48 \%$ and $23 \%$, respectively). Venom allergen 3 (Sol $i$ 3) described in the fire ant Solenopsis invicta is closely related to vespid venom antigen 5 and represents the major allergen of a series of Sol $i$ peptides identified as the most frequent cause of hypersensitivity reactions following sting from this species [18]. Amino acid sequence comparison with related Sol $i 3$ allergens from our previous work shows special signature of Sol $i$ 3-like from $T$. bicarinatum which would be relevant 


\section{A}

\section{GO: Molecular function category}

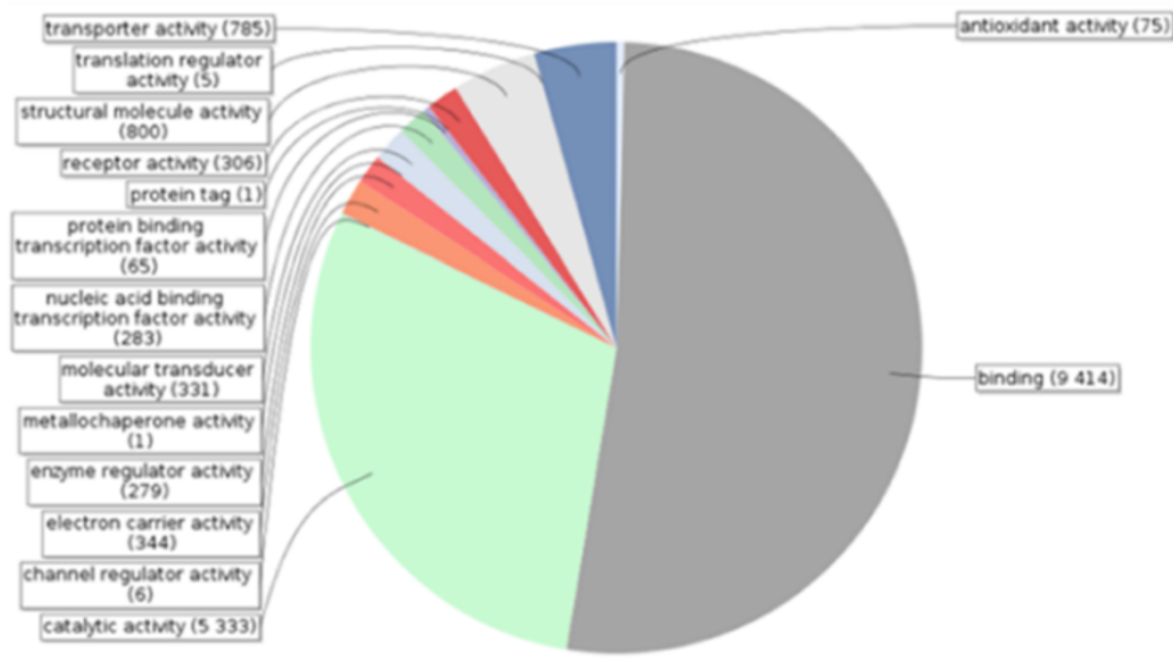

B

\section{GO: Biological process category}

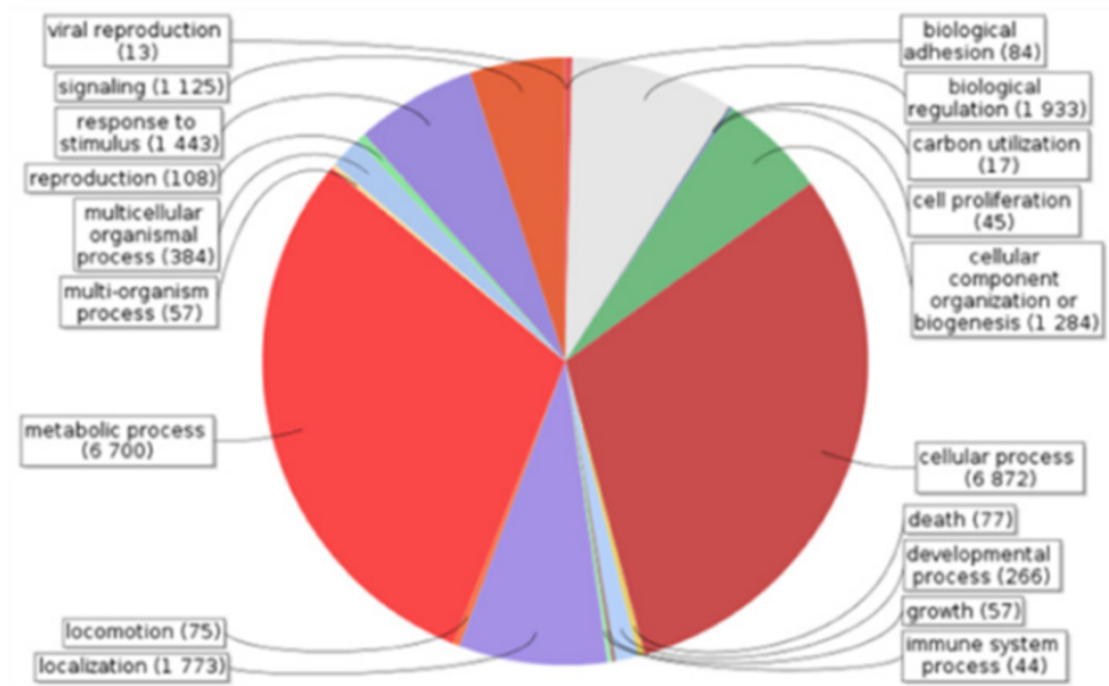

Figure 2 Gene ontology (GO) functional categorization of the generated sequences from T. bicarinatum venom glands. A: GO terms assigned in relation to the molecular function, $\mathbf{B}: \mathrm{GO}$ terms assigned according to the biological process.

in the synthesis of recombinant venom allergens for immunotherapy [13]. In addition to venom allergen 3 and 5, transcripts encoding venom acid phosphatase were detected among the major expressed toxins/allergens (13\% of the putative toxins, Figure 3). This enzyme has been found in relatively high levels in the honey bee and some social wasp venoms and recognized as potent releaser of histamine in human basophils $[19,20]$. Its biological function in Hymenoptera is however still unclear but appears to have evolved as a deterrent for vertebrate predators [5].
Members of the pilosulin-like peptides were also identified in our library. The status and features of these peptides have been discussed in our previous work where we showed that different isoforms and families of pilosulinlike sequences occur in T. bicarinatum [13]. Recently, two short peptides (named bicarinalin and peptide2) from $T$. bicarinatum venom have been characterized by de novo sequencing using mass spectrometry and Edman degradation $[21,22]$. The authors show that these mature peptides, identified among the most abundant in the venom 


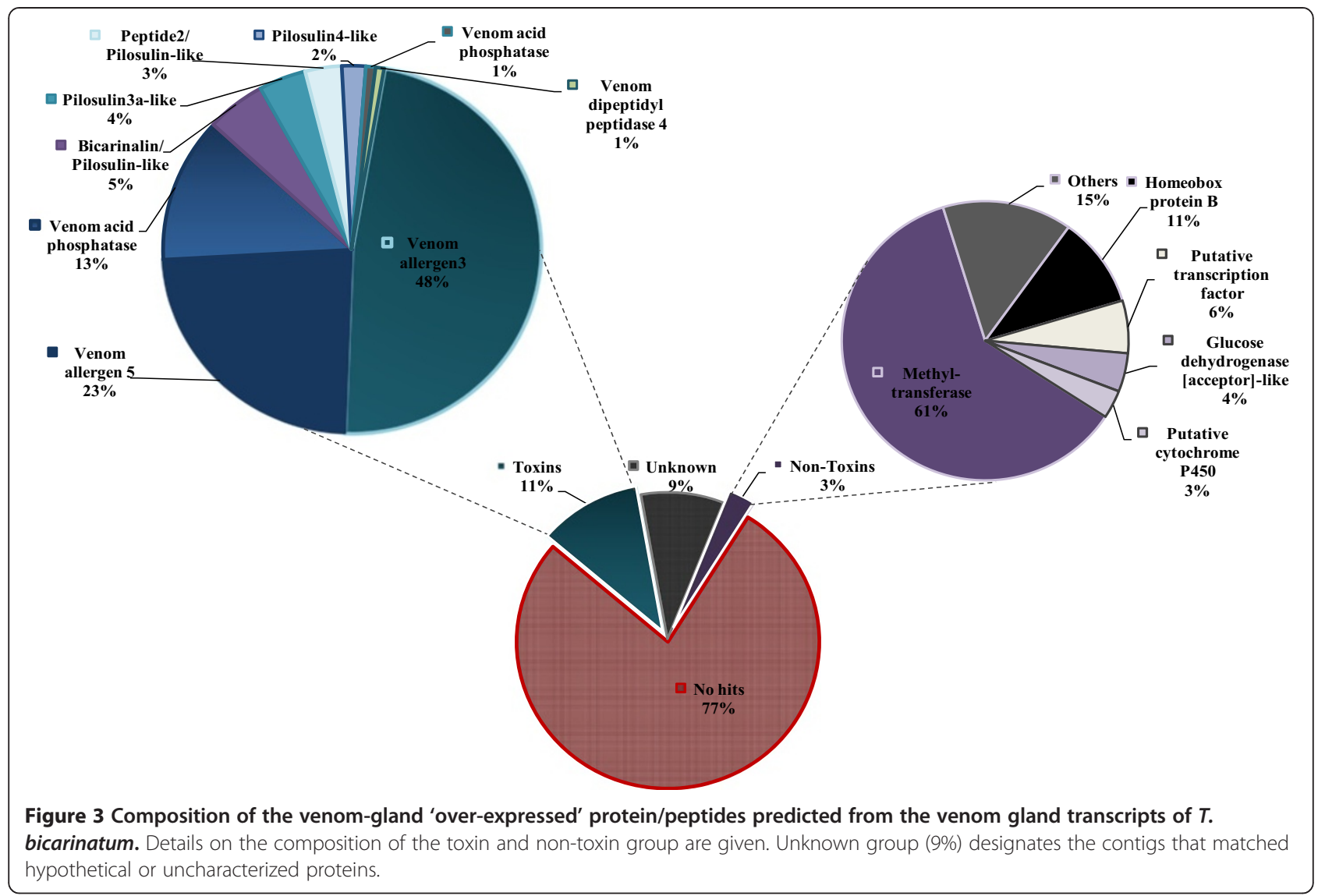

of this ant species have no homolog in the public database. In our study, we have recovered these peptides and identified their cDNA sequences (Additional file 2: Table S1). However, when we blast the whole peptide sequences predicted from the cDNA, we found that their leader sequence and propeptide have homologies with those of pilosulin sequences (Figure 4). Moreover, the bicarinalin has been shown to have a potent and broad antibacterial activity as much as pilosulins [21]. Hence, it seems that the bicarinalin and peptide 2 or the pilosulin-like forms from $T$. bicarinatum have the same features as some bioactive peptides in that they have a conserved secretory

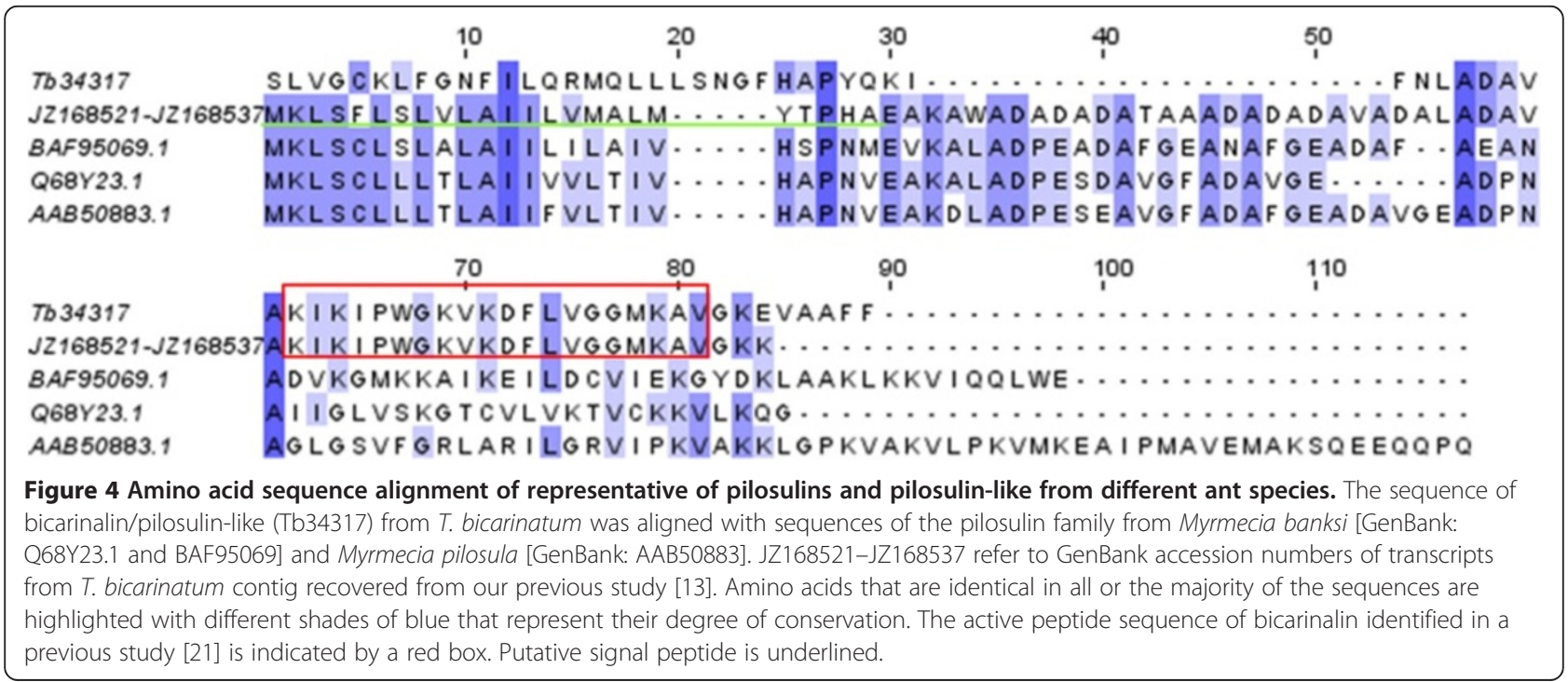


leader and propeptide sequence but differ greatly in their mature peptide $[23,24]$. This fact has been attributed to recombination as a toxin diversity-generating mechanism giving individualistic utilization of specific isoforms [25].

At a lower frequency, dipeptidylpeptidase IV has also been detected among the 'over-expressed' contig group ( $1 \%$ of the venom toxins, Figure 3 ). This enzyme is widely distributed in animal tissues and has a highly glycosylated serine protease that cleaves $\mathrm{N}$-terminal dipeptides [6]. A role in the processing of major venom compounds is ascribed to this protein [26].

In addition to the toxin found in the 'over-expressed' transcripts, possible toxins and related venom compounds were identified in this work among relatively less expressed transcripts (Additional file 2: Table S1). As some of these toxins/toxins-like have not been previously described from ant species and/or are potential pharmacological targets, a comparative study with families of related venom proteins has been investigated and discussed in respect to their amino-acid features.

\section{Venom allergen Sol II/ IV-like}

Interestingly, members of the venom allergen Sol II and the Sol IV have been identified in our study. Two contigs Tb6032 and Tb33875 (478 and 616 bp, respectively) have shown similarities with Sol II allergen described in some fire ant species. The predicted Sol II-like aminoacid sequences are 108 amino-acid long for Tb6032 and 107 for Tb33875 with a putative predicted signal peptide cleavage site for both sequences (Figure 5). The Tb6032 showed a significant identity (32\%) with the queen venom protein Sol $g$ II precursor of the species Solenopsis geminata and 32\% identity for the Sol $i$ II allergen of the species S. invicta. The Tb33875 showed 23\% and 26\% identity with these sequences, respectively. The amino-acid alignment shows a consistent conservation of the cysteine patterns and a significant conservation of the predicted signal sequence (Figure 5) which suggests the presence of Sol II venom allergen in T. bicarinatum. This finding is interesting given that up to now these venom allergens have never been identified in other animal venoms and are regarded to be specific to some Solenopsis species $[7,27]$. The main features distinguishing $T$. bicarinatum Sol II-like from its Solenopsis Sol II counterpart are the number of cysteine residues (4 against a number of 7 cysteines for Sol $i$ II) and the deletion of seven amino acid residues near the $\mathrm{N}$-terminal and 4 in the $\mathrm{C}$-terminal part.

In the same way, two transcripts of $T$. bicarinatum (Tb7500 and Tb7051) have been found similar to the venom allergen Sol IV, with consistent identity of 30 to $41 \%$. The two contigs from $T$. bicarinatum are found more related to each other (65\% of identity) than to $\mathrm{Sol}$ IV from the fire ant species. Given that the cysteine pattern is completely conserved along with three amino-acid motifs of more than 3 residues (Figure 6), this suggests that $\mathrm{Sol}$ IV-like venom allergens occur in T. bicarinatum venom. As for the Sol II, Sol IV is exclusively described from ants of the genus Solenopsis and has never been identified in any other insect venom [7,27].

Sol II and Sol IV have been described as related to each other in their amino-acid sequences but different in their expression: whereas Sol II occupy the two thirds of the protein venoms with important allergen reactivity, Sol IV represent 5 to $9 \%$ of the protein venoms with less common patient reactivity [27]. In our library, the predicted Sol IV-like has not been recorded among the 'overexpressed' transcripts, unlike venom allergens 3 and 5, but has showed higher expression rate than Sol II-like (Additional file 2: Table S1), mirroring a possible different venom dynamic expressions across ant species. Recently, a functional analysis of the $\mathrm{Sol}$ II from the species $S$. invicta emphasizes that this protein may play a role in capturing and/or transporting small hydrophobic ligands such as pheromones, odors, fatty acids, or short-living hydrophobic primers [28]. This is interesting in that some detected odor binding proteins in this study (Additional file 2: Table S1) and in our previous study [13] might be investigated as possible venom allergens [29]. 


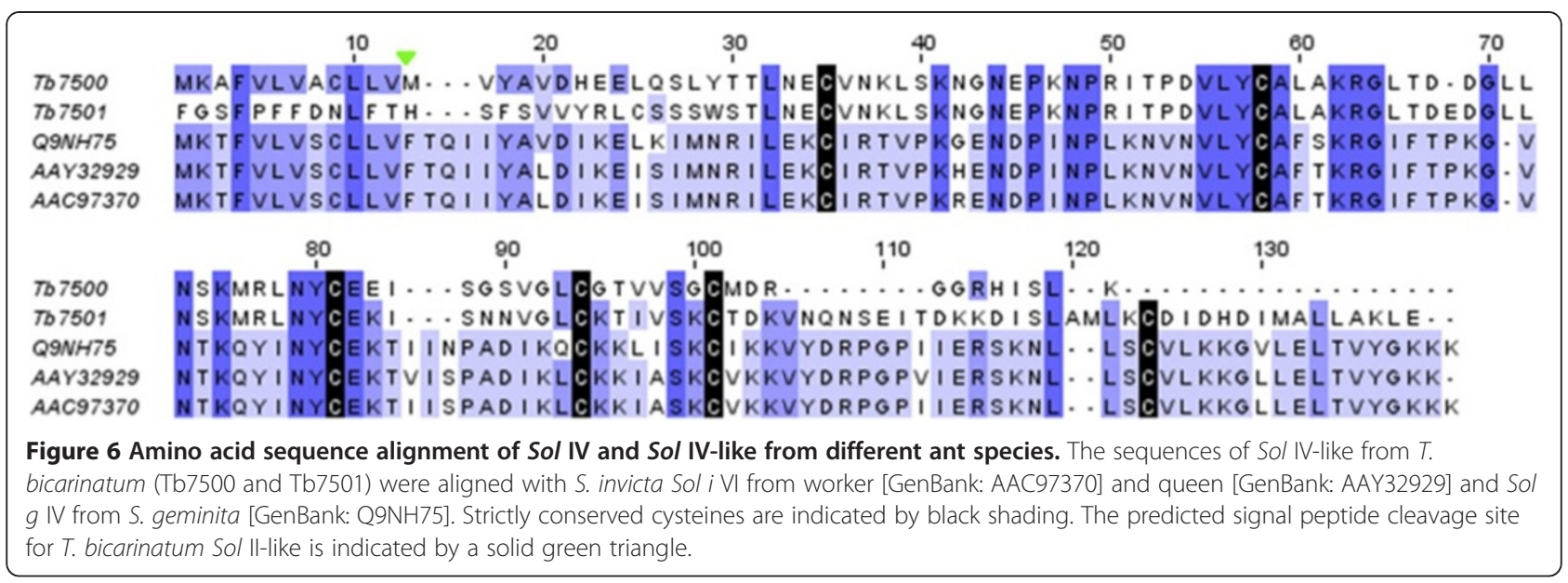

\section{T. bicarinatum PLA2-like}

Phospholipases (PLAs) are relatively common in social Hymenoptera venoms occurring in different forms and are able to disrupt the phospholipid layers of several types of biological membranes responsible of many hemolytic and neurotoxic effects (eg: [30]). In this work, five different contigs from $T$. bicarinatum matched with databases proteins of phospholipases A2 (PLA2) from Hymenoptera organisms but also from lizard and scorpion species (Figure 7A). One contig (Tb34851) was found to be closely related (91.29\% of similarities) with PLA2 previously described in the fire ant $S$. invicta. The remaining four contigs (Tb14525, Tb23564, Tb5926 and Tb21409) showed a mean range similarities of $31 \%$ for S. invicta and $33 \%$ for Apis mellifera. According to the amino-acid alignment, $T$. bicarinatum PLA2-like sequences show a high conservation of cysteine patterns and are relatively well conserved in previously identified $\mathrm{Ca}^{2+}$-binding loop region and active sites (Figure 7). The highly conserved active site residue His48 and the $\mathrm{Ca}^{2+}$-binding residues Gly32 and Asp49, available in group III PLA2 amino acid sequences [31] are also well conserved in T. bicarinatum. Taken together with the phylogenetic analysis (Figure 7B), we could assume that these PLA2-like from T. bicarinatum belong to the Group III family that have been identified in bee, lizard and scorpion venoms and characterized by low molecular mass $(13-18 \mathrm{kDa})$ and $\mathrm{Ca}^{2+}$-dependent enzyme activities [32]. It is worth to notice however a substitution of the highly conserved active site residue Tyr 125 with a phenylalanine in the contig Tb14525 of T. bicarinatum. As substitution in the active site and/or binding residues is critical and could result in inactive PLA2 [33], this substitution may affect the catalytic activity of this PLA2 T. bicarinatum form. In this work, different PLA2-like contigs were identified and the maximum-likelihood phylogeny tree supports the existence of different $T$. bicarinatum PLA2-like clusters that show a closer relationship with PLA2 from $S$. invicta than with the other aligned species from lizard and scorpion (Figure 7B). The occurrence of different PLA2 forms is in agreement with previous studies suggesting that this enzyme occurs as a series of different isoforms and/or post-translationally modified forms [34].

PLA2 was previously reported in social Hymenoptera as highly hemolytic. Its allergenic effect however was reserved to honey bee and wasp rather than ants [35]. A previous study on Hymenoptera venom PLA2 activities (including species of bee, wasp and ant) showed that each of the studied venom PLA2 exhibits lineage related specific pattern and that the chemical composition of hymenopteran venoms is biologically linked to the behavior and biology of the producing organism [36]. Indeed, a comparative study including 9 species from wasps and 9 from ants showed that phospholipase activities have generally higher levels among wasps than ants and even among ant species, differences in phospholipase concentration were also reported. While the enzyme-rich venom of the harvester ant (Pogonomyrmex badius) was reported to contain high concentrations of phospholipase A2 [37], that from the ant Myrmica ruginodis seems to lack detectable amounts of these enzyme activities [12]. In our study, PLA2-like transcripts from $T$. bicarinatum venom were not detected among the 'over-expressed' contigs suggesting a low enzyme activity. Previous study on a closer species (Tetramorium caespitum) has shown that no activity from this enzyme was detected [12], making evidence of the existence of different patterns of venom expression among and across taxa in Hymenoptera even though these venoms share a degree of similarity in activity [36].

\section{T. bicarinatum PLA1-like}

One 1284 bp long contig (Tb31783) from T. bicarinatum has been identified as venom phospholipase A1 (PLA1) and presented homologies with PLA1 of different Hymenoptera species. Figure 8 shows the aminoacid alignment of the predicted 330 amino acid sequence 


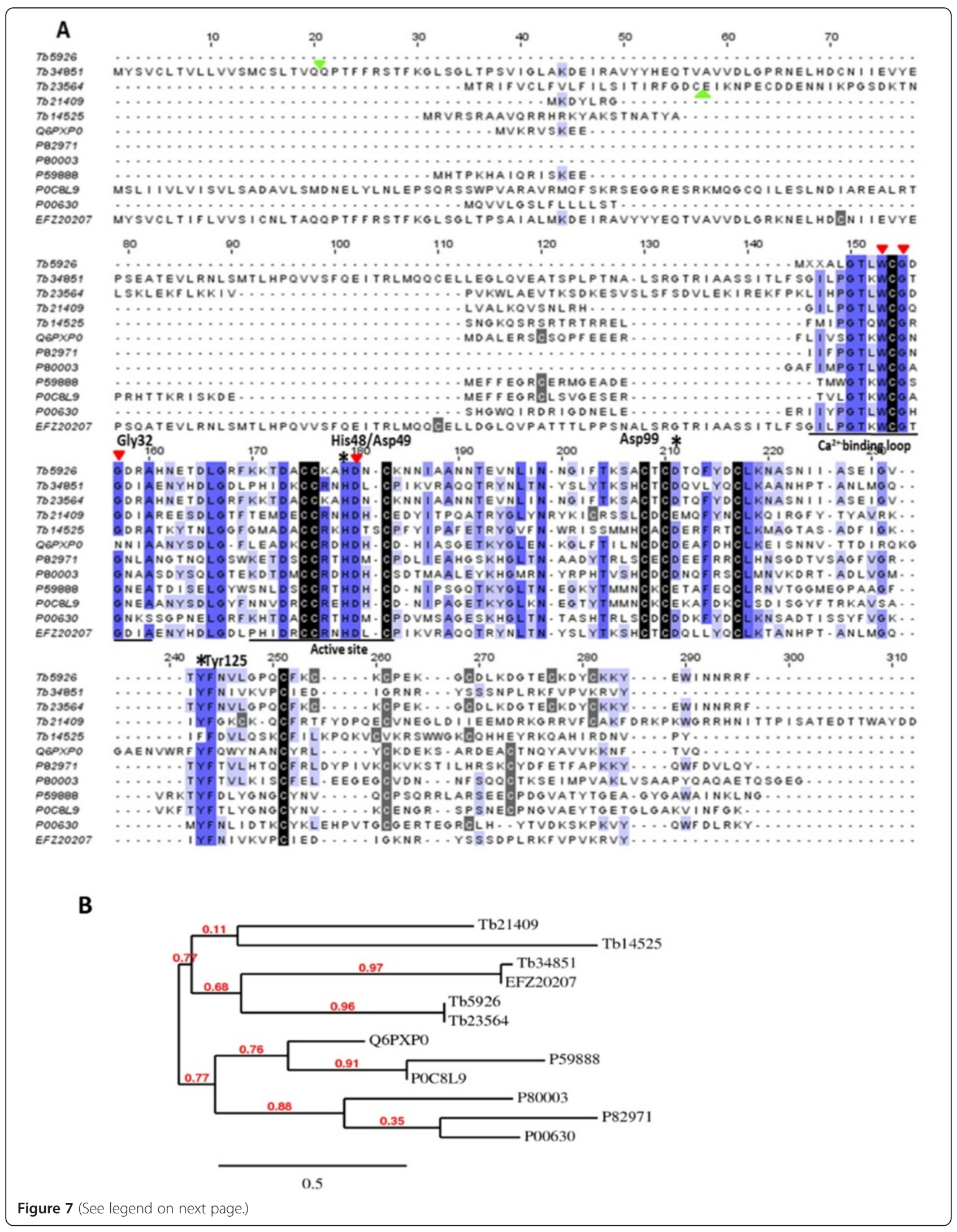


(See figure on previous page.)

Figure 7 The predicted phospholipase A2-like (PLA2) from T. bicarinatum. (A) Amino acid alignment of the sequences of PLA2-like from T. bicarinatum (Tb21409, Tb14525, Tb34851, Tb5926 and Tb23564) with PLA2 from Apis mellifera [GenBank: P00630], Bombus terrestriris [GenBank: P82971], S. invicta [GenBank: EFZ20207], Heloderma suspectum [GenBank: P80003], Hadrurus gertschi [GenBank: P0C8L9], Pandinus imperator [GenBank: P59888] and Anuroctonus phaiodactylus [GenBank: Q6PXP0]. Strictly conserved and less conserved cysteines are indicated by black and grey shading, respectively and the predicted signal peptide cleavage sites for T. bicarinatum PLA2-like is indicated by a solid green triangle. The underlined regions indicate the $\mathrm{Ca}^{2+}$ binding loop and the active site: solid red triangle, $\mathrm{Ca}^{2+}$ binding residues; ${ }^{*}$, active site residues. The numbering of the amino acid (Gly32, His48, Asp49 and Asp99) follows that of the PLA2 from bovine pancreas [31]. (B) Phylogenetic relationships of PLA2 from venomous animals based on amino acid sequence alignment. Phylogeny has been performed using the maximum likelihood method implemented in the PhyML program at www.phylogeny.fr [72]. Numbers at nodes indicate bootstrap supports based on 100 replicates. Protein codes are as described in Figure $7 \mathrm{~A}$.

from this contig with related vespid venom phospholipases and the Sol $i 1$ from S. invicta. Identity matrix shows a similarity of $31 \%$ with Sol $i 1$ from S. invicta, $36 \%$ with PLA1 from Vespa crabro, 34\% for Vespula germanica, 34\% for Vespula maculifrons and 35\% for Vespula vulgaris. The sequence alignment shows conservation of the regions that form the enzyme active site and variation in the outer regions as described in [38]. The cysteine pattern displayed by $T$. bicarinatum represents unique features comparing to the other PLA1 sequences. For example, the conserved cysteine residues at positions 217 and 222 are replaced by a deletion and a serine insertion, respectively. The missing cysteine seems to occur at the position 248 (Figure 8A). In addition, two tri-amino acid insertions and one tri-amino acid deletion occur at positions 58-60, 265-267 and 216-218, respectively. According to the phylogeny analysis, $T$. bicarinatum PLA1-like follows the Hymenoptera phylogeny and appears to be most closely related to the fire ant phospholipase than to vespid clusters with well supported bootstrap values (Figure $8 \mathrm{~B}$ ). This relatedness is in part due to the conserved cysteine residues between both species at positions 78, 165 and 365 (Figure 8A). However, the insertion of 12 residues near the C-terminal previously described in this fire ant was not detected for T. bicarinatum [38]. The PLA1 has been described in several Hymenoptera venoms and it has been shown to have no sequence similarity with other known phospholipases, but has sequence similarity with mammalian lipases [39]. Its occurrence in Hymenoptera venoms was mainly associated with tissue damages and venom diffusion [29,34].

According to the differential expression analyses of transcripts abundance, it is likely that the PLA1-like and PLA2-like identified in $T$. bicarinatum venom are not among the major allergens and venom components in this ant species. However, providing 'new' sequence features from $T$. bicarinatum venom of these enzymes could be of great importance, especially to help studies aiming to build more complete microarrays of proteins for allergy diagnosis. In addition, studies of these enzymes (especially PLA2) become useful tools for understanding ion channel structure and activity. The use of synthetic analogues from these toxins is suggested to lead to the development of new therapeutic agents and strategies for treatment of ion channel-related diseases [40]. Besides, PLA2 have been intensively described from snake venoms as antitumor targets (eg [41]) and recently it was suggested that PLA2 from honey bee venom should be tested for the preparation of cell-based cancer vaccines [42].

\section{Hyaluronidase}

A 1640 bp cDNA sequence (contig Tb32443) composed of 384 amino acid full-length ORF was identified as hyaluronidase in $T$. bicarinatum venom. The putative amino acid sequence showed a relative high similarity to hyaluronidase sequences from other ant species (a mean range similarity of $75 \%$ ) and to A. mellifera (56\%) and bombus sp. (57\%). Structural features described in venom hyaluronidases (HYALs) were highly conserved in $T$. bicarinatum, namely the four cysteine residues forming the two disulfide bridges and the active site residues (Figure 9). The -DFE- motif known to be extremely conserved and present in the active site of all of these molecules was also conserved [43] suggesting that the contig Tb32443 from T. bicarinatum encodes HYAL enzymes. HYALs are in general among the more conserved hymenopteran allergens compared to other toxins such as the venom allergen 5 and the PLA1 $[43,44]$. They were described to act as a 'venom spreading factor' in scorpion, snake, bee, and wasp venoms by hydrolyzing hyaluronic acid, a major component of the interstitial barrier, hence increasing the tissue permeability and easing venom component diffusion $[17,45]$. The expression of HYALs in Hymenoptera is very different depending on the organism. They were found in high level in the honey bee and some wasps but as a minor venom allergen in other organisms like the vespid yellow jacket [44]. In ants, enzymes with hyaluronidase activity are not described as a major component except for the Harvester ant Pogonomyrmex $[17,46]$. In our study, the generated sequences matching HYALs were about 4 times more expressed in the venom gland sequenced sample than in the ant body (Additional file 2: Table S1). Given that the venom gland library was amplified prior to sequencing, only a biochemical characterization 


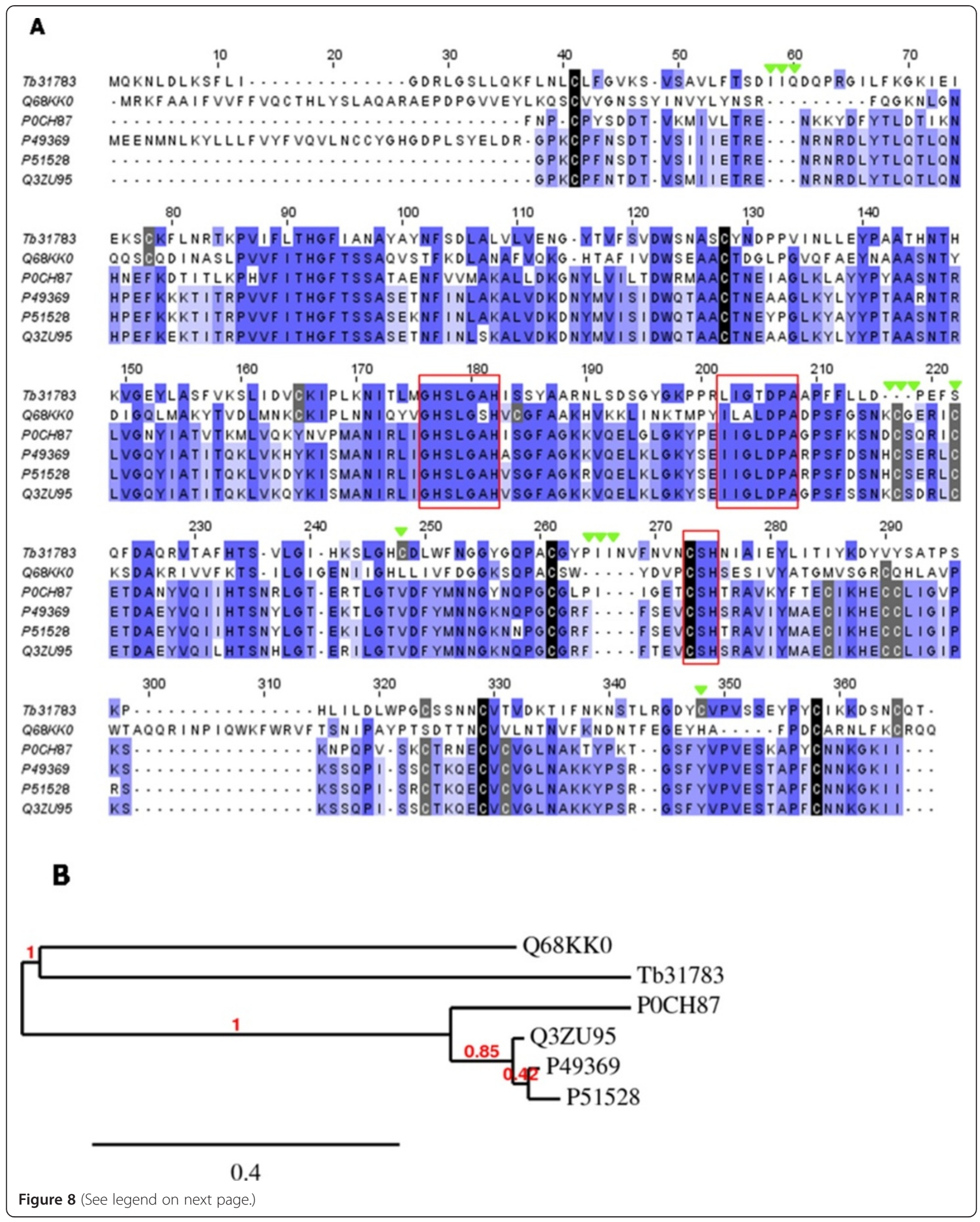


(See figure on previous page.)

Figure 8 The predicted phospholipase A1-like (PLA1) from T. bicarinatum. (A) Amino acid alignment of the sequences of PLA1-like from T. bicarinatum Tb31783 with PLA1 from S. invicta [GenBank: Q68KK0], Vespa crabro [GenBank: P0CH87], Vespula vulgaris [GenBank: P49369], Vespula maculifrons [GenBank: P51528] and Vespula germanica [GenBank: Q3ZU95]. Red boxes indicate PLA1 active sites according to [38] and green arrowheads indicate unique features for T. bicarinatum PLA1-like. Strictly conserved and non-conserved cysteine residues are shaded with black and grey, respectively. (B) Phylogenetic relationships of PLA1 from insect species based on amino acid sequence alignment. Numbers at nodes indicate bootstrap supports based on 100 replicates. Protein codes are as described in Figure 8A.

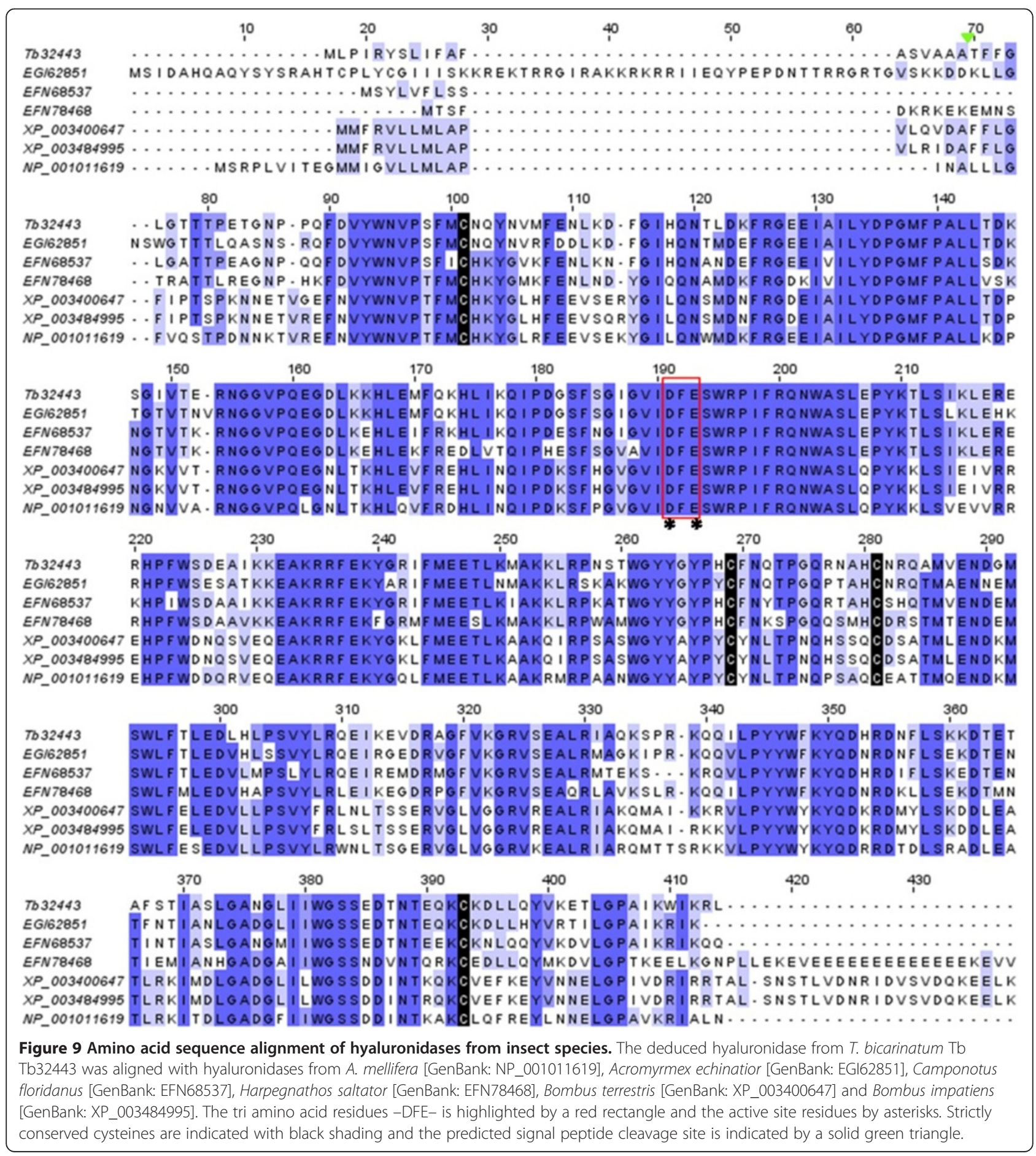


and/or a quantitative PCR should elucidate its actual expression level in T. bicarinatum.

\section{Venom serine proteases}

Two transcripts of the contig Tb23810 of 1494 bp were identified to encode Venom Serine Protesaes (VSPs). The predicted T. bicarinatum VSPs have matched with VSPs from Hymenoptera species, namely of the ant species Camponotus floridanus with $49 \%$ identity. $41 \%$ and
$40 \%$ of identity have been recorded for the bumlbee species Bombus terrestris and Bombus impatiens, respectively and $41 \%$ for the wasp Nasonia vitripennis. Consistent similarities with previously described VSP features were conserved, including the catalytic triad (Ser, His, and Asp) (Figure 10), that represents the main criterion for classification of a protein as a serine protease [47]. Serine proteases are multifunctional enzymes that play important roles in the immune response and hemostasis and are

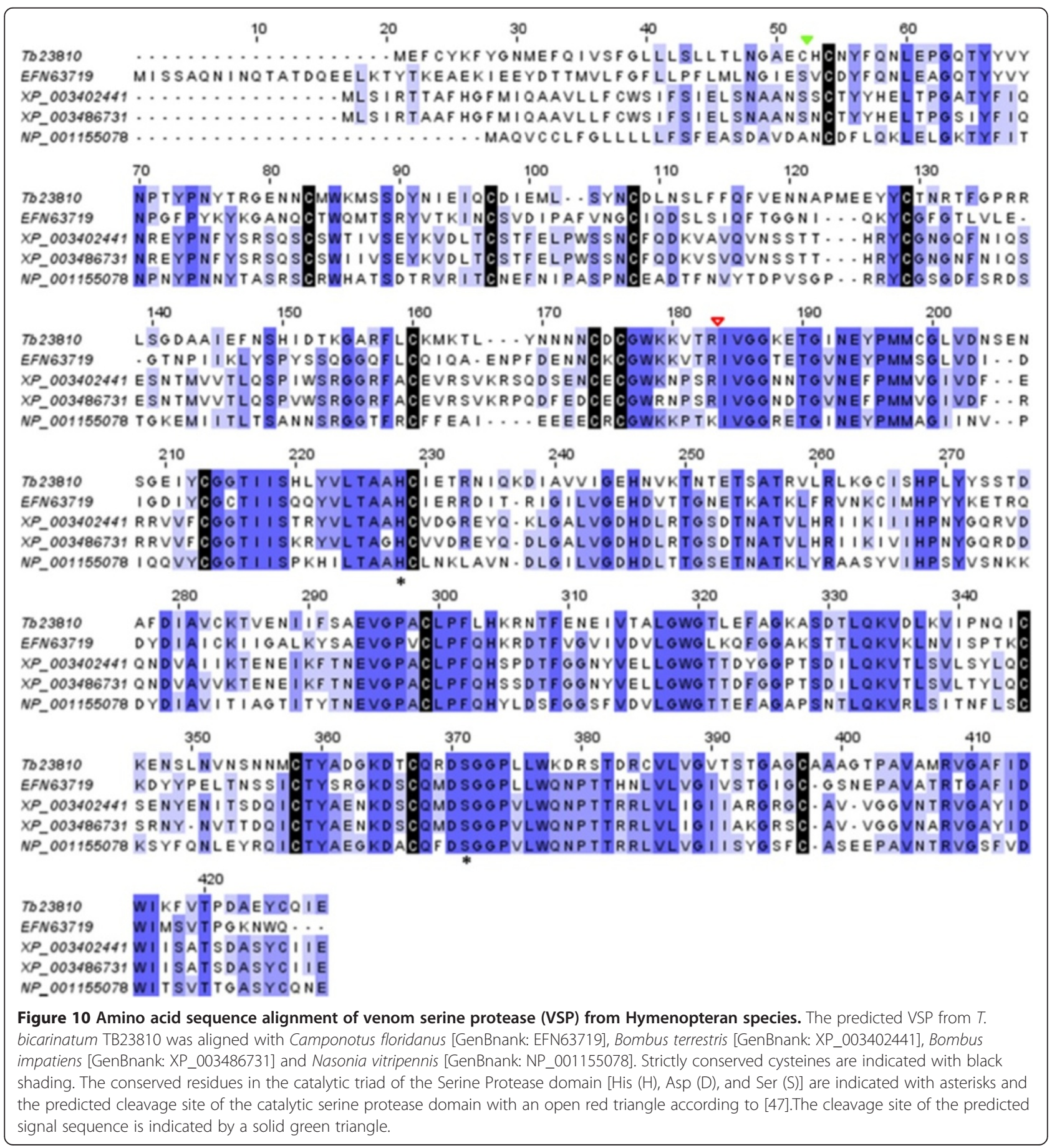


considered important allergens with significant IgE binding activity [48]. Recently, VSP from the bumblebee species Bombus ignites venom was identified as a prophenoloxidase-activating factor in insects, triggering the phenoloxidase cascade and inducing thereby a lethal melanization response in target insects; whereas in mammals, it was shown to act similarly to snake VSP, which exhibits fibrinogenolytic activity [47]. Like HYALs, VSPs were suggested to act as 'spreading factor' improving the diffusion of venom components and are likely involved in the process of their maturation or activation [49].

\section{Non hymenopteran toxins}

The blast search against $\mathrm{nr}$ database of $T$. bicarinatum contigs Tb16400 and Tb34742 of 1303 bp and 1169 bp, respectively showed similarities with waprin-like (WAPs) proteins from Hymenoptera species that were submitted to GenBank following whole genome sequencing or genome mining. In order to check the possible homologies with known WAP from other venomous species, a blast search against an in-house toxin database (see Methods section) has been achieved and has revealed homologies with snake-venom WAP. An average similarity of 33\% with the matched snake species was recorded. Domain search blast of the identified contigs against the PROSITE database [50] revealed that the predicted sequences (94 amino acid long each) contain the WAP-type 'fourdisulfide core' domain profile or whey acidic protein motif. In addition, the signature pattern of cysteine residues (CxxxxxCxxxxxCC) in the central region found in all WAP-motif proteins [51] was also identified (Figure 11), which suggests that snake-venom WAP-like are possibly expressed in T. bicarinatum venom glands. To the best of our knowledge, WAPs have never been reported from
Hymenoptera venoms. The WAP family has been described from many tissue types and organisms and has been found to have many functions, including, immunemodulation, anti-protease and anti-bacterial activities $[52,53]$. Recently, they have been described from snake venoms and from amphibian skins, each time associated with anti-microbial activities [51,54-56]. The identification of WAP from Hymenoptera venom and precisely from ant venom in this study could be of great interest regarding the vast array of its biological functions and should stimulate the investigation of their activity against a panel of micro-organisms.

In addition to WAPs-like discussed above, agatoxinlike sequences have also been detected in our analysis (Additional file 2: Table S1). Blast search against specific toxin in-house library shows a similarity of $28 \%$ and $29 \%$ of the contigs $\mathrm{Tb} 37135$ and $\mathrm{Tb} 25047$, respectively, to agatoxin sequence from the spider Agelena orientalis [GenBank accession: Q5Y4U4.1], with a completely cysteine pattern conservation (Figure 12). The amino acid sequences deduced from both contigs have an agatoxin signature of 16 residues $\mathrm{N}$-terminal signal peptide and 46 predicted mature residues. NCBI Blast of these two contigs shows consistent similarities (ranging from 68 to 91\%) with predicted agatoxin-like isoforms from the honey bee A. mellifera [GenBank accession: XP_003249808] and the bumble bee Bombus impatiens [GenBank accession: XP_003485909]. These agatoxin-like have been predicted from an automated computational analysis rather than validated from venom gland tissues. Recently, based on a combined cDNA cloning and shotgun deep sequencing approach, the spider U8 agatoxin has been recovered from the venom gland of the ant species $D$. quadriceps belonging to the Ponerinae subfamily [8]. As for T. bicarinatum

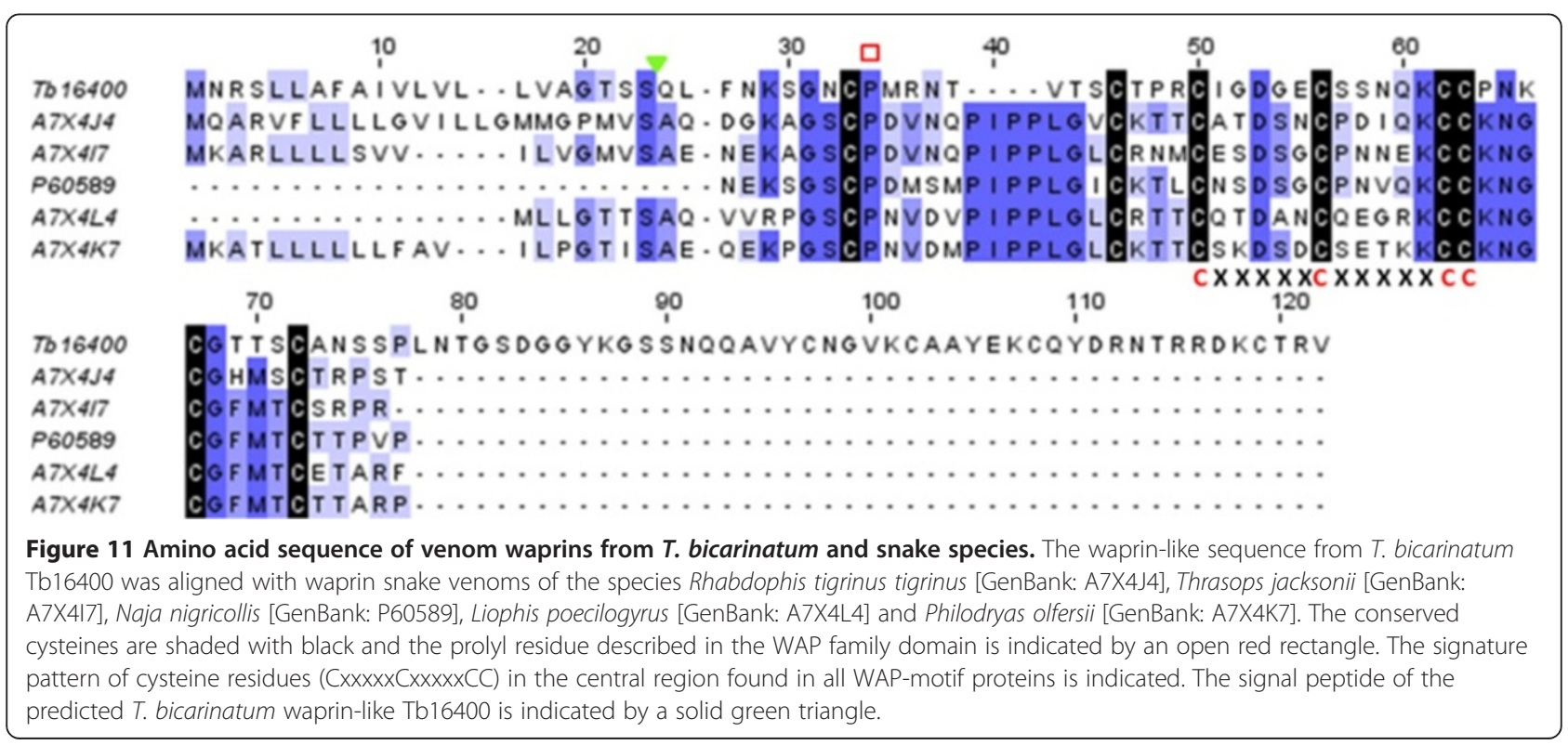




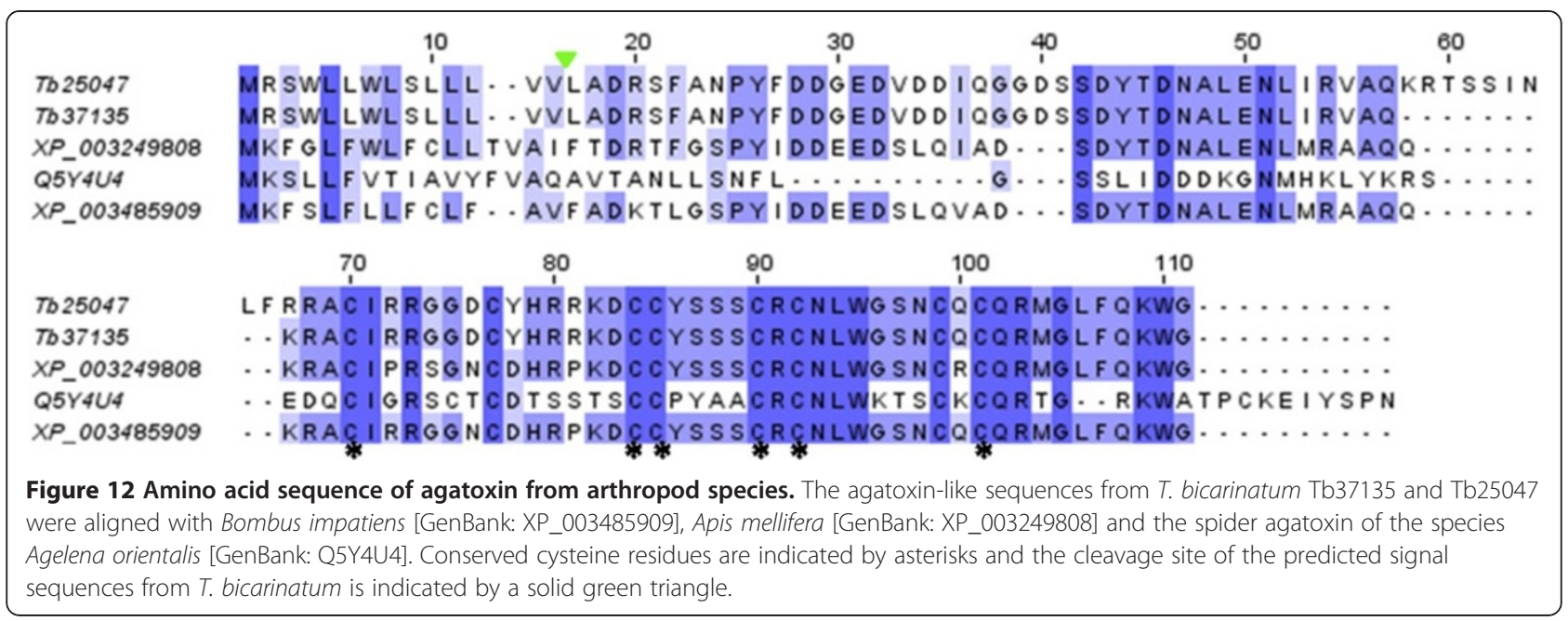

the agatoxin sequences are among the minor toxin-related components. This finding may be very useful especially for therapeutics based on new strategies for treatment of ion channel-related diseases. Indeed, agatoxins are high specific ligands for voltage-gated ion channels and are among the potential target neurotoxins that are useful tools for studying channel structure and activity [40].

\section{Other toxins}

Other putative toxins/enzymes have been detected to be expressed by $T$. bicarinatum venom glands, namely secapin, venom serine carboxylesterase, venom serine carboxypeptidase, lectizyme, disintegrin and metalloproteinase-like, chymotrypsin-like inhibitors. Details on their respective contigs, expression level are provided in (Additional file 2: Table S1). All these predicted toxin-like showed consistent similarities and homology with hymenopteran venom sequences mainly from ant species that have been submitted to public databases in the frame of whole genome sequencing. Their description in this study strengthens their existence/expression in the venom glands.

\section{No hits and putative 'new' venom toxins from $T$. bicarinatum}

In addition to the venom peptides and proteins described above, the venom gland library contained about $40 \%$ of contigs that were not identified in the public or toxin specific databases (Additional file 3: Table S2). About 3\% of these contigs with no match have been found among the arbitrary 'overexpressed' contigs where they form the most abundant group (77\%). That 'no-hits' level reflects and emphasizes the limited amount of information available for Tetramorium species and more broadly from other ants in databases. Definitely, this set of "no hit sequences" constitutes a potentially rich reservoir for the identification of novel toxins. In order to investigate the potential of these peptides as 'new' toxins, we searched for clues, such as the prediction of signal peptide and cysteine pattern. Details on statistic features of the 'no-hit' contigs are provided in (Additional file 3: Table S2). Thus, about $17 \%$ of these contigs belonging to the 'over-expressed' group have a predicted signal peptide and at least two cysteins. About $8 \%$ have signal sequences with no cysteine residues. As peptides from ant venom are more and more suggested to exhibit a defensive role against microbial pathogens, associated with prey introductions and/or ingestion [57], special investigation of $T$. bicarinatum 'no-hits' sequences was carried out using the blast search against the antimicrobial peptide database (APD). In addition, their signal peptide sequences were blasted against an in-house toxin-specific signal peptide database. Six original toxin candidates were recorded based on their relative high expression level, presence of signal peptide and on homologies (ranged between 27 to $53 \%)$ with established antimicrobial peptides. The length of the predicted mature peptide from these sequences ranged from 23 to 72 amino acids. The features of these putative novel toxins and the amino-acid alignment with their homologs from the APD are provided in (Additional file 4: Table S3). Signal peptide blast result against the in-house toxin signal peptide was not significant except for the contig Tb7117 that shows supported homology $\left(2^{\mathrm{e}-05}\right)$ with pilosulin allergens (Additional file 5: Table S4).

The identified homologs from APD are antimicrobial peptides from the venom secretion of some scorpion and spiders species or from frog skin secretions. The major expressed contig, namely the Tb34031 matches with the Charybdotoxin, an antimicrobial peptide from the yellow scorpion Leiurus quinquestriatus hebraeus with a sequence similarity of $27.94 \%$ (Additional file 4: Table S3 and Additional file 5: Table S4, respectively). 
Among the 4 cysteine residues that are present in the $T$. bicarinatum peptide, two are conserved with that of the venom scorpion peptide known to target $\mathrm{K}^{+}$channels [58]. The most important sequence homologies were recorded for the contig Tb7101 that matched with $53.57 \%$ of similarity with the ponericin W4, an antibacterial and insecticidal peptide from the venom of the ant Pachycondyla goeldii [57].

Given the homologies exhibited by the six T. bicarinatum predicted peptides identified in our study with antimicrobial peptides and their toxin-like features, it would be very interesting to characterize these peptides with proteomic approaches and test their antimicrobial potential against a panel of micro-organisms.

\section{Sequence accession numbers}

The original, unmerged sequencing reads of the venom gland library were submitted to the National Center for Biotechnology Information (NCBI) Sequence Read Archive under accession number SRR1106145. The processed and assembled data (toxin and nontoxin sequences) investigated in this study were submitted to the GenBank Transcriptome Shotgun Assembly (TSA) database. The TSA project has been deposited at DDBJ/ EMBL/GenBank under the accession GASM00000000. The version described in this paper is the first version, GASM01000000.

\section{Conclusions}

In the present study, we generated comprehensive transcriptomic data based on de novo assembly from a venom gland transcriptome of the non-model ant species $T$. bicarinatum (Hymenoptera: Formicidae). Given the limited available data from venom of this zoological group, our work greatly expanded the current knowledge of these venoms. In addition, the venom protein diversity and the presence of atypical possible venom peptides recovered in this work indicate that ant venoms are a rather complex pool with currently unknown types of venom peptides that remain to be characterized and would provide a rich unexplored resource for biomedical applications. Furthermore, data generated from sequencing of the whole ant body at transcription level from an ant species could be useful for entomologists.

\section{Methods}

\section{Ants and rearing conditions}

Polygynous colonies of the species $T$. bicarinatum were collected from Brazil (Itabuna, Bahia). The rearing conditions of the ant colonies and venom gland dissection are as described in [13]. Three hundred ant workers were sacrificed and their venom gland pooled in the same sample. They were immediately flash frozen in liquid nitrogen and stored at $-80^{\circ} \mathrm{C}$ until processed. In order to investigate differentially expressed genes, the remaining bodies of the dissected ant workers were pooled in a separate tube and immediately stored in liquid nitrogen, with prior retrieval of stomach and alimentary canal, in order to avoid contamination with genes from micro-organisms which may be present.

\section{RNA extraction and library preparation}

Total RNAs (tRNAs) from venom glands sample were isolated with RNeasy Micro Kit (Qiagen, France) including an on column DNase digestion whereas total RNAs from ant body carcasses were extracted using $400 \mu$ l of TRI reagent (Sigma) according to the manufacturer's protocol. Sequencing and cDNA library preparation were performed by Beckman Coulter Genomics services (http://www.beckmangenomics.com/). Given the very limited amount of the total RNA extracted from the venom glands $(7 \mathrm{ng} / \mu \mathrm{l})$, mRNA from this sample (sample G) was transcribed into cDNA and amplified using the Ovation RNA-Seq System V2 kit, especially applied to limited biological material (NuGEN Technologies Inc.). After cDNA fragmentation, end-repair and purification with the Agencourt ${ }^{\oplus}$ AMPure $^{\oplus}$ XP kit (Agencourt Bioscience, Beckman Coulter, San Carlos, CA, USA), TruSeq sequencing adapters (Illumina) were ligated to the cDNA fragments. Finally, the library was PCR-amplified (14 cycles) to about 20-30 ng/ $\mu \mathrm{l}$ using a high fidelity DNA polymerase. For the total RNA sample from ant carcasses (Sample F), poly (A) RNA was isolated and fragmented. First-strand cDNA synthesis was primed with an N6 randomized primer.

\section{Illumina sequencing}

Illumina TruSeq adapters were ligated to the $5^{\prime}$ and $3{ }^{\prime}$ ends of the cDNA of both samples. The cDNA was finally PCR amplified using a proof reading enzyme (Beckman genomics). For Illumina sequencing, the cDNA samples were fractionated on preparative agarose gels in the size range of 300 - $500 \mathrm{bp}$. PCR amplification was designed for TruSeq sequencing (using HiSeq2000 technology) according to the instructions of Illumina.

\section{Transcriptome assembly and analysis}

Quality of reads generated by deep sequencing was checked within the ng6 environment using fastQC program (available at http://www.bioinformatics.babraham. ac.uk/projects/fastqc/) and Burrows-Wheeler Aligner tool (BWA) to search for contamination [59]. The transcriptome de novo assembly was performed using Velvet/Oases [60]. The first step consisted of nine independent assemblies using different k-mers (k-mers for velveth: 25,31,37,43,49,55,61,65,69; parameters for velvetg: -read_trkg yes -min_contig_lgth 100 -cov_cutoff 4 and parameters for oases: -cov_cutoff 4). The raw 
transcripts files were filtered to retain only $1 \%$ of transcripts per locus with a modified version of a Perl script developed at Brown University (https://sites.google.com/ a/brown.edu/bioinformatics-in-biomed/velvet-and-oasestranscriptome). Anti-sense chimeras accidentally produced during the assembly step were cut with a home-made script. Then, independent assemblies were pooled, and duplicate/ similar transcripts build by close k-mers were removed by a cd-hit-est [61] step (parameters: - M 0 -d 0 -c 0.98) and merged by a TGICL [62] step (parameters: -1 60 -p 96 -s $100,000)$. After this assembly process, all input reads were mapped back to the set of transcripts using BWA.

\section{ORFs prediction and functional annotation}

Open Reading Frame (ORFs) were predicted by FrameDP software [63] using a home-made reference database consisting of an enriched SwissProt database [64] with venom peptides from Hymenoptera phylum. Blast search (version 2.2.26+) against nr protein and SwissProt databases was performed for ORFs annotation [65]. In addition, domain annotation was achieved with the standalone version of InterProScan [66]. Under Blast2Go software (version 2.5.0) [67], a final annotation was performed by combining Blast and InterProScan results. Furthermore, contigs with no predicted ORFs were also annotated.

In order to detect putative toxins, reviewed databases from Uniprot annotation program Tox-Prot [68] and home-made mature peptide sequences database were used. Additional in-house scripts and known tools as signalP (version 4.0) [69] have been combined to predict signal peptides.

\section{Alignment and phylogeny analysis}

Multiple sequence amino acid alignments allowing comparative study of families of related venom proteins were performed using Muscle (version 3.7) [70], then manually edited and visualized using Jalview software [71]. Phylogenetical analyses were carried out using maximum likelihood method implemented in the PhyML program at www.phylogeny.fr [72].

\section{Additional files}

\section{Additional file 1: Distribution of bacteria community in $T$.} bicarinatum according to their contig number.

Additional file 2: Table S1. Putative toxins from Tetramorium bicarinatum venoms glands.

Additional file 3: Statistics and features of 'No hit' contigs from $T$. bicarinatum venom gland library.

Additional file 4: Putative novel types of venom peptide precursors from $T$. bicarinatum.

Additional file 5: Statistical details on the putative novel venom peptides characterized from $T$. bicarinatum.

\section{Abbreviations}

ESTs: Expressed sequence tags; GO: Gene ontology; PLAs: Phospholipases; PLA2: Phospholipase A2; PLA1: Phospholipase A1; HYALs: Hyaluronidases; VSPs: Venom serine protesaes; WAPs: Waprins; APD: Antimicrobial peptide database; tRNAs: Total RNAs; BWA: Burrows-wheeler aligner; ORFs: Open reading frames.

\section{Competing interests}

The authors declare that they have no competing interests.

\section{Authors' contributions}

The project was conceived and planned by AV and WB. WB dissected the venom gland and extracted the RNA. CK, MV and CN performed the bioinformatics analysis and helped with the annotation. FD helped with the discussion and analysis of toxin features. WB wrote the manuscript and AV, FD and MV drafted it. All authors read and approved the final manuscript.

\section{Acknowledgements}

The authors thank Jérome Orivel for providing and identifying ant specimens. We are grateful to Beckman Coulter Genomics services for cDNA libraries construction and sequencing and to the GenoToul bioinformatics facility, INRA of Toulouse for providing computational resources. Funding for this work was provided to WB by a grant from the Région Midi-Pyrénées, Toulouse (France).

\section{Author details}

${ }^{1}$ Venoms and Biological Activities Laboratory, EA 4357, PRES-University of Toulouse, Jean-François Champollion University Center, Albi, France. ${ }^{2}$ Department of Pharmacology and Immunoanalysis, CEA, iBiTec-S, Gif-surYvette F-91191, France. ${ }^{3}$ The GenoToul bioinformatics platform, UR875 Biométrie et Intelligence Artificielle, INRA, Castanet-Tolosan 31326, France.

Received: 30 January 2014 Accepted: 9 September 2014 Published: 18 November 2014

\section{References}

1. Fernandes-Pedrosa MF, JF-S, YASM: An Integrated View of the Molecular Recognition and Toxinology - From Analytical Procedures to Biomedical Applications. Edited by Gandhi Rádis B. InTech; 544. Chapters published July 01, 2013 under CC BY 3.0 license doi:10.5772/3429. ISBN 978-953-51-1151-1.

2. Palma MS: Insect Venom Peptides. In Handb Biol Act Pept. San Diego: Kastin, A. Academic Press; 2006:409-417

3. Palma MS, Brochetto-Braga MR: Biochemical variability between venoms from different honey-bee (Apis mellifera) races. Comp Biochem Physiol Part C Pharmacol Toxicol Endocrinol 1993, 106:423-427.

4. Schumacher MJ, Schmidt JO, Egen NB, Dillon KA: Biochemical variability of venoms from individual European and Africanized honeybees (Apis mellifera). J Allergy Clin Immunol 1992, 90:59-65.

5. de Lima PR, Brochetto-Braga MR: Hymenoptera venom review focusing on Apis mellifera. J Venom Anim Toxins Ind Trop Dis 2003, 9:149-162.

6. De Graaf DC, Aerts M, Brunain M, Desjardins CA, Jacobs FJ, Werren JH, Devreese B: Insights into the venom composition of the ectoparasitoid wasp Nasonia vitripennis from bioinformatic and proteomic studies. Insect Mol Biol 2010, 19(Suppl 1):11-26.

7. Hoffman DR: Ant venoms. Curr Opin Allergy Clin Immunol 2010, 10:342-346.

8. Torres AFC, Huang C, Chong C-M, Leung SW, Prieto-da-Silva ARB, Havt A, Quinet YP, Martins AMC, Lee SMY, Rádis-Baptista G: Transcriptome analysis in venom gland of the predatory giant ant Dinoponera quadriceps: insights into the polypeptide toxin arsenal of hymenopterans. PLoS One 2014, 9:e87556.

9. Warrell DA, Gutiérrez JM, Calvete JJ, Williams D: New approaches \& technologies of venomics to meet the challenge of human envenoming by snakebites in India. Indian J Med Res 2013, 138:38-59.

10. Ménez A, Stöcklin R, Mebs D: "Venomics" or: the venomous systems genome project. Toxicon 2006, 47:255-259.

11. Gruber CW, Muttenthaler M: Discovery of defense- and neuropeptides in social ants by genome-mining. PLoS One 2012, 7:e32559.

12. Von Sicard NA, Candy DJ, Anderson M: The biochemical composition of venom from the pavement ant (Tetramorium caespitum L.). Toxicon 1989 27:1127-1133. 
13. Bouzid W, Klopp C, Verdenaud M, Ducancel F, Vétillard A: Profiling the venom gland transcriptome of Tetramorium bicarinatum (hymenoptera: formicidae): the first transcriptome analysis of an ant species. Toxicon 2013, 70:70-81.

14. Duan J, Xia C, Zhao G, Jia J, Kong X: Optimizing de novo common wheat transcriptome assembly using short-read RNA-Seq data. BMC Genomics 2012, 13:392.

15. Anders S, Huber W: Differential Expression of RNA-Seg Data at the Gene Level-the DESeq Package. http://www.bioconductor.org/packages/release/ bioc/vignettes/DESeq/inst/doc/DESeq.pdf.

16. Fernandes-Pedrosa Mde F, Junqueira-de-Azevedo Ide L, Gonçalves-deAndrade RM, Kobashi LS, Almeida DD, Ho PL, Tambourgi DV: Transcriptome analysis of Loxosceles laeta (Araneae, Sicariidae) spider venomous gland using expressed sequence tags. BMC Genomics 2008, 9:279.

17. Hoffman DR: Hymenoptera venom allergens. Clin Rev Allergy Immunol 2006, 30:109-128.

18. Padavattan S, Schmidt M, Hoffman DR, Marković-Housley Z: Crystal structure of the major allergen from fire ant venom, Sol i 3. J Mol Biol 2008, 383:178-185

19. Zhu J, Ye G, Hu C: Molecular cloning and characterization of acid phosphatase in venom of the endoparasitoid wasp Pteromalus puparum (Hymenoptera: Pteromalidae). Toxicon 2008, 51:1391-1399.

20. Grunwald T, Bockisch B, Spillner E, Ring J, Bredehorst R, Ollert MW: Molecular cloning and expression in insect cells of honeybee venom allergen acid phosphatase (Api m 3). J Allergy Clin Immunol 2006, 117:848-854.

21. Rifflet A, Gavalda S, Téné N, Orivel J, Leprince J, Guilhaudis L, Génin E, Vétillard $A$, Treilhou M: Identification and characterization of a novel antimicrobial peptide from the venom of the ant Tetramorium bicarinatum. Peptides 2012, 38:363-370.

22. Rifflet A: Study of Exotic Ants Venoms: Identification and Characterization of an Antimicrobial Peptide. Montpellier 1; 2012.

23. Inagaki H, Akagi M, Imai HT, Taylor RW, Wiese MD, Davies NW, Kubo T: Pilosulin 5, a novel histamine-releasing peptide of the Australian ant, Myrmecia pilosula (Jack Jumper Ant). Arch Biochem Biophys 2008, 477:411-416.

24. Vanhoye D, Bruston F, Nicolas P, Amiche M: Antimicrobial peptides from hylid and ranin frogs originated from a 150-million-year-old ancestral precursor with a conserved signal peptide but a hypermutable antimicrobial domain. Eur J Biochem 2003, 270:2068-2081.

25. Gilly WF, Richmond TA, Duda TF, Elliger C, Lebaric Z, Schulz J, Bingham JP, Sweedler JV: A diverse family of novel peptide toxins from an unusual cone snail, Conus californicus. J Exp Biol 2011, 214(Pt 1):147-161.

26. Kreil G, Haiml L, Suchanek G: Stepwise cleavage of the pro part of promelittin by dipeptidylpeptidase IV. Evidence for a new type of precursor-product conversion. Eur J Biochem 1980, 111:49-58.

27. Hoffman DR: Allergens in hymenoptera venom XXIV: the amino acid sequences of imported fire ant venom allergens Sol i II, Sol i III, and Sol i IV. J Allergy Clin Immunol 1993, 91(1 Pt 1):71-78.

28. Borer AS, Wassmann P, Schmidt M, Hoffman DR, Zhou J-J, Wright C, Schirmer T, Marković-Housley Z: Crystal structure of Sol I 2: a major allergen from fire ant venom. J Mol Biol 2012, 415:635-648.

29. Dos Santos Pinto JRA, Fox EGP, Saidemberg DM, Santos LD, da Silva Menegasso AR, Costa-Manso E, Machado EA, Bueno OC, Palma MS: Proteomic view of the venom from the fire ant Solenopsis invicta Buren. J Proteome Res 2012, 11:4643-4653.

30. Lomonte B, Angulo Y, Rufini S, Cho W, Giglio JR, Ohno M, Daniele JJ Geoghegan P, Gutiérrez JM: Comparative study of the cytolytic activity of myotoxic phospholipases A2 on mouse endothelial (tEnd) and skeletal muscle (C2C12) cells in vitro. Toxicon 1999, 37:145-158.

31. White SP, Scott DL, Otwinowski Z, Gelb MH, Sigler PB: Crystal structure of cobra-venom phospholipase $\mathrm{A} 2$ in a complex with a transition-state analogue. Science 1990, 250:1560-1563.

32. Balsinde $\mathrm{J}$ : Roles of various phospholipases $\mathrm{A} 2$ in providing lysophospholipid acceptors for fatty acid phospholipid incorporation and remodelling. Biochem J 2002, 364(Pt 3):695-702

33. Valentin $E$, Lambeau $G$ : What can venom phospholipases $A(2)$ tell us about the functional diversity of mammalian secreted phospholipases $A$ (2)? Biochimie 2000, 82:815-831.

34. Dos Santos LD, Santos KS, Pinto JRA, Dias NB, de Souza BM, dos Santos MF, Perales J, Domont GB, Castro FM, Kalil JE, Palma MS: Profiling the proteome of the venom from the social wasp Polybia paulista: a clue to understand the envenoming mechanism. J Proteome Res 2010, 9:3867-3877.

35. King TP, Spangfort MD: Structure and biology of stinging insect venom allergens. Int Arch Allergy Immunol 2000, 123:99-106.

36. Zalat S, Schmidt J, Moawad TI: Lipase and phospholipase activities of Hymenoptera venoms (wasps and ants). Egypt J Biol 2005, 5:138-147.

37. Schmidt JO, Blum MS: A harvester ant venom: chemistry and pharmacology. Science 1978, 200:1064-1066.

38. Hoffman DR, Sakell RH, Schmidt M: Sol i 1, the phospholipase allergen of imported fire ant venom. J Allergy Clin Immunol 2005, 115:611-616.

39. Soldatova L, Kochoumian L, King TP: Sequence similarity of a hornet (D. maculata) venom allergen phospholipase A1 with mammalian lipases. FEBS Lett 1993, 320:145-149.

40. Pringos E, Vignes M, Martinez J, Rolland V: Peptide neurotoxins that affect voltage-gated calcium channels: a close-up on $\omega$-agatoxins. Toxins (Basel) 2011, 3:17-42.

41. Araya C, Lomonte B: Antitumor effects of cationic synthetic peptides derived from Lys49 phospholipase A2 homologues of snake venoms. Cell Biol Int 2007, 31:263-268.

42. Almunia C, Bretaudeau M, Held G, Babon A, Marchetti C, Castelli FA, Ménez A, Maillere B, Gillet D: Bee venom phospholipase A2, a good "chauffeur" for delivering tumor antigen to the $\mathrm{MHC}$ I and MHC II peptide-loading compartments of the dendritic cells: the case of NY-ESO-1. PLoS One 2013, 8:e67645.

43. Justo Jacomini DL, Campos Pereira FD, Aparecido dos Santos Pinto JR, dos Santos LD, da Silva Neto AJ, Giratto DT, Palma MS, de Lima Zollner R, Brochetto Braga MR: Hyaluronidase from the venom of the social wasp Polybia paulista (Hymenoptera, Vespidae): cloning, structural modeling, purification, and immunological analysis. Toxicon 2013, 64:70-80.

44. Jin C, Focke M, Léonard R, Jarisch R, Altmann F, Hemmer W: Reassessing the role of hyaluronidase in yellow jacket venom allergy. J Allergy Clin Immunol 2010, 125:184-190. e1.

45. Kemparaju K, Girish KS: Snake venom hyaluronidase: a therapeutic target. Cell Biochem Funct 2006, 24:7-12.

46. Hink WF, Pappas PW, Jaworski DC: Partial biochemical characterization of venom from the ant, Pseudomyrmex triplarinus. Toxicon 1994, 32:763-772.

47. Choo YM, Lee KS, Yoon HJ, Kim BY, Sohn MR, Roh JY, Je YH, Kim NJ, Kim I, Woo SD, Sohn HD, Jin BR: Dual function of a bee venom serine protease: prophenoloxidase-activating factor in arthropods and fibrin (ogen) olytic enzyme in mammals. PLoS One 2010, 5:e10393.

48. Hoffman DR, Jacobson RS: Allergens in hymenoptera venom. XXVII: bumblebee venom allergy and allergens. J Allergy Clin Immunol 1996, 97:812-821.

49. Lima PRM DE, Brochetto-Braga MR, Chaud-netto J: Proteolytic activity of africanized honeybee (Apis mellifera: hymenoptera, apidae) venom. J Venom Anim Toxins 2000, 6:64-76

50. Falquet L, Pagni M, Bucher $P$, Hulo N, Sigrist CJA, Hofmann K, Bairoch A: The PROSITE database, its status in 2002. Nucleic Acids Res 2002, 30:235-238.

51. Ali MF, Lips KR, Knoop FC, Fritzsch B, Miller C, Conlon JM: Antimicrobial peptides and protease inhibitors in the skin secretions of the crawfish frog, Rana areolata. Biochim Biophys Acta 2002, 1601:55-63.

52. Hagiwara K, Kikuchi T, Endo Y, Huqun, Usui K, Takahashi M, Shibata N, Kusakabe T, Xin H, Hoshi S, Miki M, Inooka N, Tokue Y, Nukiwa T: Mouse SWAM1 and SWAM2 are antibacterial proteins composed of a single whey acidic protein motif. J Immunol 2003, 170:1973-1979.

53. Wiedow O, Schröder JM, Gregory H, Young JA, Christophers E: Elafin: an elastase-specific inhibitor of human skin. Purification, characterization, and complete amino acid sequence. J Bio/ Chem 1990, 265:14791-14795.

54. St Pierre L, Earl ST, Filippovich I, Sorokina N, Masci PP, De Jersey J, Lavin MF: Common evolution of waprin and kunitz-like toxin families in Australian venomous snakes. Cell Mol Life Sci 2008, 65:4039-4054

55. Nair DG, Fry BG, Alewood P, Kumar PP, Kini RM: Antimicrobial activity of omwaprin, a new member of the waprin family of snake venom proteins. Biochem J 2007, 402:93-104

56. Liu D, Wang Y, Wei L, Ye H, Liu H, Wang L, Liu R, Li D, Lai R: Snake venom-like waprin from the frog of Ceratophrys calcarata contains antimicrobial function. Gene 2013, 514:99-104.

57. Orivel J, Redeker V, Le Caer JP, Krier F, Revol-Junelles AM, Longeon A, Chaffotte A, Dejean A, Rossier J: Ponericins, new antibacterial and insecticidal peptides from the venom of the ant Pachycondyla goeldii. J Biol Chem 2001, 276:17823-17829. 
58. Yount NY, Yeaman MR: Multidimensional signatures in antimicrobial peptides. Proc Natl Acad Sci U S A 2004, 101:7363-7368.

59. Mariette J, Escudié F, Allias N, Salin G, Noirot C, Thomas S, Klopp C: NG6: integrated next generation sequencing storage and processing environment. BMC Genomics 2012, 13:462.

60. Li H, Durbin R: Fast and accurate short read alignment with BurrowsWheeler transform. Bioinformatics 2009, 25:1754-1760.

61. Schulz MH, Zerbino DR, Vingron M, Birney E: Oases: robust de novo RNA-seq assembly across the dynamic range of expression levels. Bioinformatics 2012, 28:1086-1092.

62. Li W, Godzik A: Cd-hit: a fast program for clustering and comparing large sets of protein or nucleotide sequences. Bioinformatics 2006, 22:1658-1659.

63. Gouzy J, Carrere S, Schiex T: FrameDP: sensitive peptide detection on noisy matured sequences. Bioinformatics 2009, 25:670-671.

64. Consortium T: The universal protein resource (UniProt) in 2010. Nucleic Acids Res 2010, 38(Database issue):D142-D148.

65. Camacho C, Coulouris G, Avagyan V, Ma N, Papadopoulos J, Bealer K, Madden TL: BLAST+: architecture and applications. BMC Bioinformatics 2009, 10:421.

66. Zdobnov EM, Apweiler R: InterProScan-an integration platform for the signature-recognition methods in InterPro. Bioinformatics 2001, 17:847-848.

67. Conesa A, Götz S, García-Gómez JM, Terol J, Talón M, Robles M: Blast2GO: a universal tool for annotation, visualization and analysis in functional genomics research. Bioinformatics 2005, 21:3674-3676.

68. Jungo F, Bairoch A: Tox-Prot, the toxin protein annotation program of the Swiss-Prot protein knowledgebase. Toxicon 2005, 45:293-301.

69. Bendtsen JD, Nielsen H, von Heijne G, Brunak S: Improved prediction of signal peptides: SignalP 3.0. J Mol Biol 2004, 340:783-795.

70. Edgar RC: MUSCLE: multiple sequence alignment with high accuracy and high throughput. Nucleic Acids Res 2004, 32:1792-1797.

71. Waterhouse AM, Procter JB, Martin DMA, Clamp M, Barton GJ: Jalview version 2-a multiple sequence alignment editor and analysis workbench. Bioinformatics 2009, 25:1189-1191.

72. Dereeper A, Guignon V, Blanc G, Audic S, Buffet S, Chevenet F, Dufayard J-F, Guindon S, Lefort V, Lescot M, Claverie J-M, Gascuel O: Phylogeny.fr: robust phylogenetic analysis for the non-specialist. Nucleic Acids Res 2008, 36(Web Server issue):W465-W469.

doi:10.1186/1471-2164-15-987

Cite this article as: Bouzid et al:: De Novo sequencing and transcriptome analysis for Tetramorium bicarinatum: a comprehensive venom gland transcriptome analysis from an ant species. BMC Genomics 2014 15:987.

\section{Submit your next manuscript to BioMed Central and take full advantage of:}

- Convenient online submission

- Thorough peer review

- No space constraints or color figure charges

- Immediate publication on acceptance

- Inclusion in PubMed, CAS, Scopus and Google Scholar

- Research which is freely available for redistribution

Submit your manuscript at www.biomedcentral.com/submit
Biomed Central 